\title{
Long-term morbidities in stroke survivors: a prospective multicenter study of Thai stroke rehabilitation registry
}

Vilai Kuptniratsaikul ${ }^{1 *}$, Apichana Kovindha ${ }^{2}$, Sumalee Suethanapornkul ${ }^{3}$, Nuttaset Manimmanakorn ${ }^{4}$ and Yingsumal Archongka ${ }^{5}$

\begin{abstract}
Background: Stroke-related complications are barriers to patients' recovery leading to increasing morbidity, mortality, and health care costs, decreasing patient's quality of life. The purpose of this study was to quantify incidence and risk factors of stroke-related complications during the first year after discharge from rehabilitation ward.

Methods: A prospective observational study was conducted in nine tertiary-care rehabilitation centers. We evaluated the incidence of morbidities during the first year after stroke, including musculoskeletal pain, neuropathic pain, pneumonia, deep vein thrombosis (DVT), pressure ulcer, spasticity, shoulder subluxation, joint contracture, dysphagia, urinary incontinence, anxiety and depression. The complications at discharge and at month-12 were compared using the McNemar test. Univariate analysis and multiple logistic regression analysis by forward stepwise method were used to determine factors predicting the complications at month-12.
\end{abstract}

Results: Two hundred and fourteen from 327 patients (65.4\%) were included. The age was $62.1 \pm 12.5$ years, and 57.9\% were male. In $76.8 \%$ of the patients at least one complication was found during the first year after stroke. Those complications were musculoskeletal pain (50.7\%), shoulder subluxation (29.3\%), depression (21.2\%), spasticity (18.3\%), joint contracture (15.7\%) and urinary incontinence (14.4\%). Other complications less than 5\% were dysphagia (3.5\%), pressure ulcer (2.6\%), infection (1.5\%), and neuropathic pain (3.0\%). Nearly $60 \%$ of patients with complications at discharge still had the same complaints after one year. Only $7.6 \%$ were without any complication. Morbidity was significantly associated with age and type of stroke. Using multiple logistic regression analysis, age and physical complications at discharge were significant risk factors for physical and psychological morbidities after stroke respectively $(\mathrm{OR}=2.1,95 \% \mathrm{Cl} 1.2,3.7 ; \mathrm{OR}=3.1,95 \% \mathrm{Cl} 1.3,7.1)$.

Conclusion: Long-term complications are common in stroke survivors. More than three-fourths of the patients developed at least one during the first year after rehabilitation. Strategies to prevent complications should be concerned especially on musculoskeletal pain which was the most common complaint. Physical complications at discharge period associated with psychological complications at 1 year followed up. More attention should be emphasized on patients age older than 60 years who were the major risk group for developing such complications.

Keywords: Stroke, Morbidity, Registry, Multi-center study, Pain, Depression

\footnotetext{
* Correspondence: vilai.kup@mahidol.ac.th

${ }^{1}$ Rehabilitation Medicine Department, Faculty of Medicine Siriraj Hospital,

Mahidol University, 2 Prannok Rd, Bangkok 10700, Thailand

Full list of author information is available at the end of the article
} 


\section{Background}

Stroke is one of the most public health concerns worldwide as it is the leading cause of disability in the elderlies [1]. It limits the patients' physical, psychological, and social functions. In-patient rehabilitation enhances the chances for functional recovery, greater independence and life satisfaction [2]. Although rehabilitation can improve self-care and ambulatory functions, the patients are still vulnerable to various stroke morbidities [3].

Stroke-related complications are barriers to patients' recovery, increasing morbidity, mortality, and health care costs while decreasing quality of life [4]. In 2008, we established Thai Stroke Rehabilitation Registry (TSRR), the first multi-center and hospital-based registry of rehabilitation for stroke patients in Thailand, and reported the stroke-related complications in 327 stroke patients found during their hospitalization for initial rehabilitation [5]. We found that $71.0 \%$ developed at least one complication during such period; the findings were similar to those of other studies [6,7]. Since medical complications obstruct health recovery after stroke and lead to poor outcomes [8], the information regarding types and frequency of stroke-related complications would be helpful for providing appropriate management to the patients. To date, there is no long-term follow-up data of morbidities in Thai stroke survivors. Therefore, we conducted a prospective observational study to assess the incidence and risk factors of morbidities in stroke survivors during the first year after discharge from rehabilitation ward.

\section{Methods}

The present study was a multi-center, prospective observational study in nine tertiary care medical institutes in Thailand. It was conducted in accordance with the ethical principles stated in the most recent version of the Declaration of Helsinki. The study protocols were approved by the Institutional Review Board of 9 tertiary hospitals including Institutional Review Board, Faculty of Medicine Siriraj Hospital, Mahidol University (reference number 316/2006), Ethical Clearance Committee on Human Rights Related to Research involving Human Subjects, Faculty of Medicine Ramathibodi Hospital, Mahidol University (reference number 061/2007), Institutional Review Board, Faculty of Medicine, Chulalongkorn University (reference number 033/2007), Institutional Review Board, Royal Thai Army Medical Department (reference number 1248/2006), Research Ethics Committee of Chiang Mai University, Faculty of Medicine (reference number 002/2007), Khon Kean University Ethics Committee for Human Research (reference number: 4.2.04:1/ 2007), Institutional Review Board, Faculty of Medicine, Prince of Songkla University (reference number 369-003/ 2007), Prasat Neurological Institutional Review Board and Ethic Committee (reference number 021/2007) and Ethical Committee of Sirindhorn National Medical Rehabilitation Center (reference number 003/2007). Written informed consent was obtained from patients for the permission to use their medical information for the present report.

Participants with a diagnosis of newly stroke registered to previous study (TSRR) from 2008 [9] were included, while patients whose follow-up data were not available due to any reasons, for example unable to contact, living in rural area, or being unable to come to the hospitals, were excluded from the present study. This study used a standardized structured record form to collect the medical data one record per patient. The principal investigator of each institute evaluated the outcomes in when patients came to be followed up at the hospital.

The severity of the disease was assessed by Barthel Index (BI) [10]. It was categorized to be 5 grades of very severely disabled (score 0-4), severely disabled (score 5-9), moderately disabled (score 10-14), mild disabled (score 15-19) and independently (score 20). Stroke-related complications included musculoskeletal pain, neuropathic pain, pneumonia, deep vein thrombosis (DVT), pressure ulcer, spasticity, shoulder subluxation, joint contracture, dysphagia, urinary incontinence, anxiety and depression. Pressure ulcers were categorized into 4 stages; stage 1: nonblanchable erythema not resolved within 30 minutes; stage 2: partial thickness loss of skin involving epidermis; stage 3: full thickness destruction through dermis into subcutaneous tissue; stage 4: deep tissue destruction into fascia, muscle, bone or joint [11]. Spasticity was evaluated at the elbow flexor and knee flexor using the Modified Ashworth Scale (MAS) [12]. Degree of spasticity was recorded only moderate, or severe $(\mathrm{MAS} \geq 3)$. Shoulder subluxation was diagnosed if the distance between the acromion process and the head of the humerus was wider than one finger breadth [13]. Anxiety and depression were evaluated using the Thai version of the Hospital Anxiety and Depression Scale (HADS) [14]. It had 14 items which 7 items of odd number represents anxiety while the other 7 with even number represents depressive mood. The score ranges from $0-21$ for each dimension. The patients who had a score $\geq 11$ of each part were considered as having clinical anxiety or depression. Additionally, factors associated with stroke-related complications during the first year were also analyzed.

\section{Statistical analysis}

Data were analyzed using computerized software, PASW statistic 18. Demographic data were presented in number and percentage for categorical data or in mean and standard deviation (SD) for continuous data. The rates of complications at discharge and at month-12 were compared using the McNemar test. Univariate analysis was used to determine factors predicting the complications at month- 12 . 
Factors with a p-value less than 0.20 were subsequently analyzed using multiple logistic regression analysis by forward stepwise method. A p-value less than 0.05 was considered statistically significant.

\section{Results}

During the study period there were 327 patients in the TSRR. The median duration from onset to admission interval (OAI) for rehabilitation was 24 days [9]. Only 214 patients $(65.4 \%)$ could be followed up for at least one year, whereas 8 (2.4\%) died, 3 withdrew and 102 lost to follow up. Concerning the severity of participants evaluated by BI, $42.8 \%$ was mild, $17.9 \%$ moderately, $13.8 \%$ severely to very severely disabled and only $25.5 \%$ was independently [15]. The baseline characteristics of 214 patients are presented in Table 1. Their mean age was $62.1 \pm 12.5$ years and $57.9 \%$ were males. Most patients were married (70.6\%) and almost all of them (96.7\%) had family support. More than $70 \%$ had brain infarction. The major underlying medical diseases were hypertension (75.7\%), followed by dyslipidemia (55.1\%), diabetes mellitus (29.0\%), cardiac diseases (18.7\%), and previous stroke (15.0\%). Of 214 patients, $137(64.0 \%)$ had at least 2 underlying diseases including diabetes mellitus, hypertension, dyslipidemia, previous stroke, or atrial fibrillation.

Among 214 patients, 198 had completed data in case record form, therefore 152 (76.8\%) patients had at least one complication including musculoskeletal pain, shoulder subluxation, anxiety, depression, incontinence, spasticity,

\section{Table 1 Baseline characteristics of 214 stroke patients}

\begin{tabular}{|c|c|}
\hline Demographic data & Mean \pm SD or $\mathrm{N}(\%)$ \\
\hline \multirow[t]{2}{*}{ Age (yrs) } & $62.1 \pm 12.5$ \\
\hline & (range 21-93) \\
\hline Sex: male & $124(57.9)$ \\
\hline Marital status: married & $151(70.6)$ \\
\hline Type of pathology: Infarction & $155(72.4)$ \\
\hline Presence of family support & $207(96.7)$ \\
\hline \multicolumn{2}{|l|}{ Underlying diseases: } \\
\hline - Hypertension & $162(75.7)$ \\
\hline - Dyslipidemia & $118(55.1)$ \\
\hline - Diabetes mellitus & $62(29.0)$ \\
\hline - Cardiac diseases (CAD, Ml, AF, LVH) & $40(18.7)$ \\
\hline - Previous stroke/TIA & $32(15.0)$ \\
\hline $\begin{array}{l}\text { - Others (AVM, Carotid stenosis, Diabetic } \\
\text { retinopathy, Hypothyroid, Parkinson, Seizure, } \\
\text { Spinal stenosis) }\end{array}$ & $41(19.2)$ \\
\hline Underlying diseases: $\geq 2$ diseases ${ }^{\#}$ & $137(64.0)$ \\
\hline \multicolumn{2}{|c|}{$\begin{array}{l}\text { Note: } \mathrm{CAD}=\text { coronary artery disease, } \mathrm{MI}=\text { myocardial infarction, } \mathrm{AF}=\text { atrial } \\
\text { fibrillation, } \mathrm{LVH}=\text { left ventricular hypertrophy, } \mathrm{TIA}=\text { transient ischemic attack, } \\
\mathrm{AVM}=\text { atherovenous malformation. } \\
\text { \# Include diabetes mellitus, hypertension, dyslipidemia, previous stroke, } \\
\text { atrial fibrillation. }\end{array}$} \\
\hline
\end{tabular}

dysphagia, pressure ulcer, infection and joint contracture. Concerning the number of complications occurred, the percentage of patients who had 1, 2, 3, 4 and $\geq 5$ complications were $30.8,20.7,12.1,7.1$ and 6.1 respectively.

Table 2 presented the common stroke related complications at month-12 compared to those at discharge period. For the overall complications, nearly $60 \%$ of patients with complications at discharge still had the same complaints after one year. Among the patients who did not have complication at discharge, $20 \%$ subsequently developed complications during the first year; whereas only $7.6 \%$ were without any complication. The top five complications found during the first year of stroke were musculoskeletal pain (50.7\%), shoulder subluxation (29.3\%), depression (21.2\%), spasticity (18.3\%) and joint contracture (15.7\%). Among the musculoskeletal pain, shoulder was the most common site with an incidence of $33.9 \%$. Approximately one-third of patients with musculoskeletal pain did not have such complication at discharge. This was also true for $25 \%$ of shoulder pain or shoulder subluxation, $10.5 \%$ of anxiety, and $16.5 \%$ of depression. Joint contracture was not presented at discharge, but it developed later during the follow-up period. The common sites of contracture were shoulder, ankle, and knee joints. Urinary incontinence was found in $14.4 \%$ of the patients. Other complications less than $5 \%$ were dysphagia (3.5\%), pressure ulcer $(2.6 \%)$, infection (1.5\%), and neuropathic pain (3.0\%).

Table 3 shows the review of post-stroke complications during the first year after stroke among various studies. Table 4 reveals the factors associating with stroke-related complications after discharge from rehabilitation unit. Univariate analysis demonstrated that age and pathology of stroke, were significant factors associating with the presence of complications during the first year of stroke; whereas other factors including sex, onset to admission interval (OAI), family support, anxiety and depression scores, Barthel score at discharge, and LOS were not. Multiple logistic regression analysis by forward stepwise method demonstrated that age older than 60 years was the only important factor associating with the complications during the first year after discharge (OR $=2.1,95 \% \mathrm{CI}=1.1,4.1)$.

Table 5 analyzed the factors associated to physical complications mainly of pain in all aspects, while Table 6 analyzed those with psychological complications including anxiety and depression. After using multiple logistic analysis, we found that age still was the only factor associated to physical complications at 1 year $(\mathrm{OR}=2.1,95 \%$ $\mathrm{CI}=1.2$, 3.7). In addition, physical complication at discharge was related to psychological complications at 1 year. $(\mathrm{OR}=3.1,95 \% \mathrm{CI}=1.3,7.1)$.

\section{Discussion}

The complications after stroke were common problems causing lost of self care functions, psychological impact, 
Table 2 The common stroke-related complications at month-12 compared at discharge period

\begin{tabular}{lccc}
\hline $\begin{array}{l}\text { Complications } \\
\text { at discharge }\end{array}$ & Number & $\begin{array}{c}\text { Complications } \\
\text { at month-12 }\end{array}$ & p-value $^{\#}$ \\
\cline { 2 - 2 } & Yes No &
\end{tabular}

Overall complications

198

Yes

No

$\begin{array}{ll}113(57.1 \%) & 31(15.6 \%) \\ 39(19.7 \%) & 15(7.6 \%)\end{array}$

0.403

Musculoskeletal pain

Yes

No

Shoulder pain

Yes

No

Neuropathic pain

Yes

No

Limb spasticity ${ }^{\mathrm{a}}$ :

$\geq$ grade 3

Yes

No

Shoulder subluxation ${ }^{\text {b: }}$

Yes

No

Anxiety: score $\geq 11$

Yes

No

Depression: score $\geq 11$

Yes

No

Dysphagia

Yes

No

Urinary incontinence

Yes

No

Pressure ulcer

Yes

No

DVT

Yes

No

Infection

Yes

No

Joint contracture ${ }^{c}$

- Shoulder
201

$\begin{array}{ll}35(17.4 \%) & 19(9.5 \%) \\ 67(33.3 \%) & 80(39.8 \%)\end{array}$

201

$\begin{array}{ccc}17(8.5 \%) & 22(10.9 \%) & 0.001^{*} \\ 51(25.4 \%) & 111(55.2 \%)\end{array}$

196

$$
0(0.0 \%)
$$$$
7(3.6 \%)
$$

1.000

$6(3.0 \%) \quad 183(93.4 \%)$

191

$$
\begin{array}{cc}
4(2.1 \%) & 19(10.0 \%) \\
31(16.2 \%) & 137(71.7 \%)
\end{array}
$$

199

$$
9(4.7 \%) \quad 14(7.3 \%) \quad<0.001^{*}
$$$$
47(24.6 \%) \quad 121(63.4 \%)
$$

171

$$
\begin{array}{ccc}
2(1.2 \%) & 6(3.5 \%) & 0.023^{*} \\
18(10.5 \%) & 145(84.8 \%) &
\end{array}
$$

170

$$
\begin{array}{ccc}
8(4.7 \%) & 17(10.0 \%) & 0.135 \\
28(16.5 \%) & 117(68.8 \%) &
\end{array}
$$

$$
3(1.5 \%) \quad 26(12.9 \%)<0.001^{*}
$$$$
4(2.0 \%) \quad 169 \text { (83.6\%) }
$$

202

$$
8(4.0 \%) \quad 38(18.8 \%) \quad 0.036^{*}
$$$$
21(10.4 \%) \quad 135(66.8 \%)
$$

196

$\begin{array}{ccc}- & 5(2.6 \%) & 1.000 \\ 5(2.6 \%) & 186(94.8 \%) & \\ - & 1(0.5 \%) & 1.000 \\ - & 196(99.5 \%) & \end{array}$

199

$\begin{array}{ccc}1(0.5 \%) & 26(13.1 \%) & <0.001^{*} \\ 2(1.0 \%) & 170(85.4 \%) & \\ 30(15.7 \%) & 161(84.3 \%) \\ 20(10.5 \%) & 171(89.5 \%)\end{array}$

Table 2 The common stroke-related complications at month-12 compared at discharge period (Continued)

$\begin{array}{lcc}\text { - Hip } & 3(1.6 \%) & 188(98.4 \%) \\ \text { - Knee } & 8(4.2 \%) & 183(95.8 \%) \\ \text { - Ankle } & 10(5.2 \%) & 181(94.8 \%)\end{array}$

\# Data were analyzed using McNemar Chi-square test.

${ }^{a}$ Spasticity was evaluated by the Modified Ashworth Scale ${ }^{12}$ Grade 1: slight increase in muscle tone, minimal resistance at the end of range of motion when the affected part is moved. Grade 1+: slight increase in muscle tone, minimal resistance throughout the remainder (less than half) of the range of motion. Grade 2: more marked increase in muscle tone through most of the ROM. Grade 3: considerable increase in muscle tone, passive movement difficult. Grade 4: affected part rigid in flexion or extension.

b Shoulder subluxation was categorized according to the World Health Organization's International Classification of Functioning, Disability and Health, $2001 .^{13}$ Grade 1 (mild): severity of symptoms less than $25 \%$. Grade 2 (moderate): symptoms less than $50 \%$. Grade 3 (severe): symptoms more than $50 \%$. Grade 4 (very severe): symptoms more than $95 \%$.

c There was no contracture at discharge.

social disability and decreased quality of life. In our present study, $76.8 \%$ of the patients had at least one complication; the incidence of which was comparable to that of Pinedo and de la Villa (81\%) [16]. Within the first year of stroke, half of our patients suffered from the musculoskeletal pain; our incidence was similar to that of Sackley et al. (55\%) [17]. From our previous report,

\begin{tabular}{|c|c|c|c|c|}
\hline \multirow[t]{2}{*}{ Complications } & $\begin{array}{l}\text { Langhorne } \\
\quad(n=180)\end{array}$ & $\begin{array}{c}\text { Pinedo }^{16} \\
(n=73)\end{array}$ & $\begin{array}{l}\text { Sackley }^{17} \\
(n=122)\end{array}$ & $\begin{array}{c}\text { Our study } \\
(n=198)\end{array}$ \\
\hline & 2000 & 2001 & 2008 & 2013 \\
\hline $\begin{array}{l}\text { Having at least } \\
\text { one complications }\end{array}$ & N/A & 81 & N/A & 76.8 \\
\hline $\begin{array}{l}\text { Musculoskeletal } \\
\text { pain }\end{array}$ & 35 & - & 55 & 50.7 \\
\hline Shoulder pain & 11 & 40 & 52 & 33.9 \\
\hline $\begin{array}{l}\text { Shoulder } \\
\text { subluxation }\end{array}$ & - & - & - & 29.3 \\
\hline Depression & 43 & - & 50 & 21.2 \\
\hline Spasticity (MAS $\geq 3$ ) & - & - & - & 18.3 \\
\hline Joint contracture & - & 23 & 60 & 15.7 \\
\hline Urinary incontinence & 23 & - & - & 14.4 \\
\hline Anxiety & 44 & - & - & 11.7 \\
\hline Pressure ulcer & 8 & - & 22 & 2.6 \\
\hline Fall & 49 & - & 73 & - \\
\hline RSD of arm & - & 15 & - & - \\
\hline
\end{tabular}
musculoskeletal pain was also the top rank complication during the initial rehabilitation phase [5]. Hansen et al. found that $45.8 \%$ of stroke patients had post-stroke pain during the first 6 months, and $16.4 \%$ were shoulder pain [18]. We found only one-third (33.9\%) of participants suffered with shoulder pain which was similar to the study from Lund Stroke Register [19]. They revealed that

Table 3 The rate of post-stroke complications (percent) among various studies 
Table 4 Factors associating with stroke-related complications developed during the first year after stroke using univariate analysis and multiple logistic regression analysis

\begin{tabular}{|c|c|c|c|c|}
\hline Factors & Complications $^{@}$ & No complication & Odds ratio $(95 \% \mathrm{Cl})$ & p-value ${ }^{\#}$ \\
\hline \multicolumn{5}{|l|}{$\operatorname{Sex}(n=198)$} \\
\hline Male & $81(72.3 \%)$ & $31(27.7 \%)$ & - & 0.091 \\
\hline Female & $71(82.6 \%)$ & $15(17.4 \%)$ & & \\
\hline \multicolumn{5}{|l|}{ Age $(n=198)$} \\
\hline$<60$ years & $58(69.0 \%)$ & $26(31.0 \%)$ & 1 & $0.027^{*}$ \\
\hline$\geq 60$ years & $94(82.5 \%)$ & $20(17.5 \%)$ & $2.1(1.1,4.1)$ & \\
\hline \multicolumn{5}{|l|}{$\mathrm{OAI}^{\mathrm{a}}(\mathrm{n}=198)$} \\
\hline$<3$ months & $124(78.0 \%)$ & $35(22.0 \%)$ & - & 0.412 \\
\hline$\geq 3$ months & $28(71.8 \%)$ & $11(28.2 \%)$ & & \\
\hline \multicolumn{5}{|c|}{ Pathology $(n=198)$} \\
\hline Hemorrhage & $36(66.7 \%)$ & $18(33.3 \%)$ & - & $0.039^{*}$ \\
\hline Infarction & $116(80.6 \%)$ & $28(19.4 \%)$ & & \\
\hline \multicolumn{5}{|c|}{ Psychological complications (anxiety/depression) at DC $(n=168)$} \\
\hline$<11$ & $107(78.1 \%)$ & $30(21.9 \%)$ & - & 0.396 \\
\hline$\geq 11$ & $22(71.0 \%)$ & $9(29.0 \%)$ & & \\
\hline \multicolumn{5}{|c|}{ Physical complications at DC $(n=198)$} \\
\hline No & $50(71.4 \%)$ & $20(28.6 \%)$ & - & 0.188 \\
\hline Yes & $102(79.7 \%)$ & $26(20.3 \%)$ & & \\
\hline \multicolumn{5}{|c|}{ Family support $(n=198)$} \\
\hline No & $1(33.3 \%)$ & $2(66.7 \%)$ & - & 0.135 \\
\hline Yes & $151(77.4 \%)$ & $44(22.6 \%)$ & & \\
\hline \multicolumn{5}{|l|}{$\operatorname{LOS}^{b}(n=198)$} \\
\hline$<1$ months & $88(76.5 \%)$ & $27(23.5 \%)$ & - & 0.923 \\
\hline$\geq 1$ months & $64(77.1 \%)$ & 19 (22.9\%) & & \\
\hline \multicolumn{5}{|c|}{ Bl score ${ }^{c}$ at DC $(n=198)$} \\
\hline$<15$ & $84(79.2 \%)$ & $22(20.8 \%)$ & - & 0.376 \\
\hline$\geq 15$ & 68 (73.9\%) & $24(26.1 \%)$ & & \\
\hline
\end{tabular}

@ Complications included musculoskeletal pain, neuropathic pain, pneumonia, deep vein thrombosis (DVT), pressure ulcer, spasticity, shoulder subluxation, joint contracture, dysphagia, urinary incontinence, anxiety and depression.

"Data were analyzed using Chi-square test or Fisher's exact test.

${ }^{a} \mathrm{OAI}=$ Onset to admission interval, ${ }^{\mathrm{b}} \mathrm{LOS}=$ Length of stay, ${ }^{\mathrm{c}} \mathrm{BI}=$ Barthel Index $<15$ means moderately to severely disabled, $\geq 15$ means mildly disabled to independently, DC = discharge.

"Statistical significance.

almost one third of 327 patients developed shoulder pain during the first year. Kocabas et al. found the relationship between shoulder pain and subluxation, loss of range of motion, spasticity of shoulder muscles and muscle strength. Therefore, they recommended performing range of motion (ROM) exercise of the glenohumeral joint, strengthening shoulder muscles and reduction of spasticity in stroke patients in order to prevent shoulder pain after stroke [20].

In addition, shoulder subluxation was another common complication after stroke (29.3\%). Among those subluxation group, one-fourth (24.6\%) was the new complication found at month-12. Shoulder subluxation associated with shoulder pain and poor upper extremity function [21]. We also found the prevalence of stroke subluxation during rehabilitation period of $37.3 \%$ which was mild to moderate degree [5]. It should be treated during acute stage with better result. According to Ottawa panel evidence-based clinical practice guideline, treatment of shoulder subluxation was one of the recommendations for post stroke rehabilitation [22]. There were evidences of the efficacy of additional functional electrical stimulation (FES) to the supraspinatus and posterior deltoid muscles are more beneficial than conventional treatment $[23,24]$.

Joint contracture was found in $15.7 \%$ of our patients during the first year which was less than other studies $[16,17]$. This may due to Sackley et al. studied in nursing 
Table 5 Factors associating with physical complications developed during the first year after stroke using univariate analysis and multiple logistic regression analysis

\begin{tabular}{|c|c|c|c|c|}
\hline Factors & Physical complications $^{@}$ & No physical complication & Odds ratio $(95 \% \mathrm{Cl})$ & p-value \\
\hline \multicolumn{5}{|l|}{$\operatorname{Sex}(n=198)$} \\
\hline Male & $51(45.9 \%)$ & $60(54.1 \%)$ & - & 0.053 \\
\hline Female & $52(59.8 \%)$ & $35(40.2 \%)$ & & \\
\hline \multicolumn{5}{|l|}{ Age $(n=198)$} \\
\hline$<60$ years & $35(41.7 \%)$ & $49(58.3 \%)$ & 1 & $0.012^{*}$ \\
\hline$\geq 60$ years & $68(59.6 \%)$ & $46(40.4 \%)$ & $2.1(1.2,3.7)$ & \\
\hline \multicolumn{5}{|l|}{$\mathrm{OAl}^{\mathrm{a}}(\mathrm{n}=198)$} \\
\hline$<3$ months & 87 (54.0\%) & $74(46.0 \%)$ & - & 0.236 \\
\hline$\geq 3$ months & $16(43.2 \%)$ & $21(56.8 \%)$ & & \\
\hline \multicolumn{5}{|c|}{ Pathology $(n=198)$} \\
\hline Hemorrhage & $21(38.9 \%)$ & $33(61.1 \%)$ & - & $0.024^{*}$ \\
\hline Infarction & $82(56.9 \%)$ & $62(43.1 \%)$ & & \\
\hline \multicolumn{5}{|c|}{ Psychological complications (anxiety/depression) at DC $(n=166)$} \\
\hline$<11$ & $74(54.8 \%)$ & $61(45.2 \%)$ & - & 0.331 \\
\hline$\geq 11$ & $14(45.2 \%)$ & $17(54.8 \%)$ & & \\
\hline \multicolumn{5}{|c|}{ Physical complications at DC $(n=198)$} \\
\hline No & $32(43.8 \%)$ & $41(56.2 \%)$ & - & 0.078 \\
\hline Yes & $71(56.8 \%)$ & $54(43.2 \%)$ & & \\
\hline \multicolumn{5}{|c|}{ Family support $(n=198)$} \\
\hline No & - & $3(100.0 \%)$ & - & 0.109 \\
\hline Yes & $103(52.8 \%)$ & $92(47.2 \%)$ & & \\
\hline \multicolumn{5}{|l|}{$\operatorname{LOS}^{b}(n=198)$} \\
\hline$<1$ months & $62(53.0 \%)$ & $55(47.0 \%)$ & - & 0.742 \\
\hline$\geq 1$ months & $41(50.6 \%)$ & 40 (49.4\%) & & \\
\hline \multicolumn{5}{|c|}{ Barthel score ${ }^{c}$ at $D C(n=198)$} \\
\hline$<15$ & $58(56.3 \%)$ & $49(51.7 \%)$ & - & 0.504 \\
\hline$\geq 15$ & $45(43.7 \%)$ & $46(48.3 \%)$ & & \\
\hline
\end{tabular}

\footnotetext{
$@$ Physical complications mainly represented pain in all aspects.

"Data were analyzed using Chi-square test or Fisher's exact test.

${ }^{\mathrm{a}} \mathrm{OAI}=$ Onset to admission interval, ${ }^{\mathrm{b}} \mathrm{LOS}=$ Length of stay, ${ }^{\mathrm{C}} \mathrm{BI}=$ Barthel Index $<15$ means moderately to severely disabled, $\geq 15$ means mildly disabled to independently, DC = discharge.

Statistical significance.
}

homes' subjects which were more disabled than ours. Comparing to the study of Pinedo \& de la Villa [16], our prevalence was close to them (23\%). This may because their setting was rehabilitation ward as ours. However, we supposed from the results that stroke patients had inadequate ROM exercise at home. Therefore, therapists should emphasize the need of adequate ROM exercise to the patients and their caregiver in order to prevent this complication.

Table 3 shows the review of post-stroke complications during the first year after stroke among various studies. Compared with other studies in Table 3, the prevalence of almost all complications during the first year after stroke in our study was less. This might be owing to the difference in study designs, patient characteristics, and definitions of the complications. For example, Sackley et al. studied stroke patients with severe disability and functional dependence in nursing homes [17]. Therefore, the prevalence of complications in their study was quite higher than others. Another reason may be from some studies assumed the frequency of complications at follow up period from patients' report or relatives, so their prevalence may be underestimated or overestimated [20]. Nevertheless, these studies including ours support that post-stroke complications are quite common; therefore rehabilitation programs to prevent complications should be emphasized for recognition among therapists. Early detection and treatment of complications would result in good outcome. 
Table 6 Factors associating with psychological complications (anxiety/depression) developed during the first year after stroke using univariate analysis and multiple logistic regression analysis

\begin{tabular}{|c|c|c|c|c|}
\hline Factors & Psychological complications $^{@}$ & No psychological complication & Odds ratio $(95 \% \mathrm{Cl})$ & p-value ${ }^{\#}$ \\
\hline \multicolumn{5}{|l|}{$\operatorname{Sex}(n=200)$} \\
\hline Male & $28(23.9 \%)$ & $89(76.1 \%)$ & - & 0.565 \\
\hline Female & $17(20.5 \%)$ & $66(79.5 \%)$ & & \\
\hline \multicolumn{5}{|l|}{ Age $(n=200)$} \\
\hline$<60$ years & $20(22.7 \%)$ & $68(77.3 \%)$ & - & 0.946 \\
\hline$\geq 60$ years & $25(22.3 \%)$ & $87(77.7 \%)$ & & \\
\hline \multicolumn{5}{|l|}{$O A I^{a}(n=200)$} \\
\hline$<3$ months & $34(21.3 \%)$ & $126(78.7 \%)$ & - & 0.397 \\
\hline$\geq 3$ months & $11(27.5 \%)$ & $29(72.5 \%)$ & & \\
\hline \multicolumn{5}{|l|}{$\begin{array}{l}\text { Pathology } \\
(n=200)\end{array}$} \\
\hline Hemorrhage & $8(14.3 \%)$ & $48(85.7 \%)$ & - & 0.083 \\
\hline Infarction & $37(25.7 \%)$ & $107(74.3 \%)$ & & \\
\hline \multicolumn{5}{|c|}{$\begin{array}{l}\text { Psychological complications (anxiety/depression) } \\
\text { at DC }(n=170)\end{array}$} \\
\hline$<11$ & $29(20.6 \%)$ & $112(79.4 \%)$ & - & 0.105 \\
\hline$\geq 11$ & $10(34.5 \%)$ & $19(65.5 \%)$ & & \\
\hline \multicolumn{5}{|c|}{ Physical complications at DC $(n=200)$} \\
\hline No & $8(11.4 \%)$ & $62(88.6 \%)$ & 1 & $0.006^{*}$ \\
\hline Yes & $37(28.5 \%)$ & $93(71.5 \%)$ & $3.1(1.3,7.1)$ & \\
\hline \multicolumn{5}{|c|}{ Family support $(n=200)$} \\
\hline No & - & $3(100.0 \%)$ & - & 1.000 \\
\hline Yes & $45(22.8 \%)$ & $152(77.2 \%)$ & & \\
\hline \multicolumn{5}{|l|}{$\operatorname{LOS}^{b}(n=200)$} \\
\hline$<1$ months & $24(20.2 \%)$ & $95(79.8 \%)$ & - & 0.338 \\
\hline$\geq 1$ months & $21(25.9 \%)$ & $60(74.1 \%)$ & & \\
\hline \multicolumn{5}{|c|}{ Bl score ${ }^{c}$ at DC $(n=200)$} \\
\hline$<15$ & $26(57.8 \%)$ & $78(50.2 \%)$ & - & 0.378 \\
\hline$\geq 15$ & $19(42.2 \%)$ & $77(49.8 \%)$ & & \\
\hline
\end{tabular}

$@$ Psychological complications represented anxiety and depression.

"Data were analyzed using Chi-square test or Fisher's exact test.

${ }^{\mathrm{a}} \mathrm{OAI}=$ Onset to admission interval, ${ }^{\mathrm{b}}$ LOS = Length of stay, ${ }^{\mathrm{c}} \mathrm{BI}=$ Barthel Index $<15$ means moderately to severely disabled, $\geq 15$ means mildly disabled to independently, DC = discharge.

"Statistical significance.

Previous studies demonstrated various risk factors associating with the development of complications during the first year of stroke. These factors included patient dependency, duration after stroke, and low scores on the Barthel Index $[17,25]$. Our previous study reported that duration of disease $\geq 1$ month (adjusted OR $=2.12$, 95\% $\mathrm{CI}=1.07-4.17$ ), length of stay $>21$ days (adjusted $\mathrm{OR}=$ $2.36,95 \% \mathrm{CI}=1.26-4.43$ ), and anxiety score at admission $\geq 11$ (adjusted OR $=6.87,95 \% \mathrm{CI}=2.45-19.29$ ) were associated with stroke related complications during the initial rehabilitation phase [5]. When the patients were followed up longer, age was the only important factor associating with complications developed during the first year. Khan et al. reported that older age was one of the independent predictors of poor functional outcome of stroke survivors with adjust $\mathrm{OR}=2.1$ [26]. As age was non-modifiable factor, the only intervention that we can provide is to promote exercise in order to maintain or improve physical health of the patients. A meta-analysis on physical activity and stroke risk found that highly physically active persons had a $27 \%$ lower risk of stroke incidence or mortality than did persons of low activity [27]. Therefore, strategies to encourage our stroke patients to maintain physical activity or continue exercise at home should be performed in order to prevent complications after stroke. 
Another factor related to psychological complications including anxiety and depression, found in this study was physical complications at discharge period. Anxiety and depression were common in stroke patients. Previous studies found that these complications were present in almost half of the patients $[17,25,28]$. The lower incidence of such complications in our present study might be owing to the different diagnostic tools used in our study. For example, Sackley et al. chose the score $\geq 7$ of the HADS to be considered of depressed mood [17], but we used score $\geq 11$ to be diagnosis of depression. Langhorne et al. [25] assessed depression by interviewing with questions "do you often feel sad or depressed?" and "do you often feel anxious or agitated?", while we used standardized questionnaire (HADS) with definite cut-off score. Therefore, the prevalence of anxiety and depression in their study was quite higher than ours. Although anxiety and depression were common after stroke, our study revealed that stroke patients who had physical complications at discharge had 3 times risk for psychological complications. Physicians should pay attention to these common complications because the earlier the diagnosis and treatment were provided, the better the outcome of treatment would be gained.

The limitation of our study was high proportion of drop off patients (31.2\%). This was because nearly 40 percent of subjects lived in rural area and the transportation system in our country was inconvenient for disabled. In addition, almost all subjects had poor socioeconomic status. Therefore, it is difficult for them and their relatives to come to the hospital in urban area. Our study did not have enough funds to visit them at their homes. Therefore, our results can infer only stroke patients who can come to be followed up at the hospital.

\section{Conclusions}

Long-term complications are common in stroke survivors. The top five complications were musculoskeletal pain, shoulder subluxation, depression, spasticity and joint contracture. More than three-fourths of the patients developed at least one during the first year after rehabilitation. Strategies to prevent complications should be concerned especially on musculoskeletal pain which was the most common complaint. More attention should be emphasized on patients age older than 60 years who were the major risk group for developing such complications.

\section{Competing interests}

All of the authors declare no financial competing interests.

\section{Authors' contributions}

VK, AK, SS, NM and YA participated in conception, data analysis and interpretation of data. VK and AK conceived the study, involved in drafting the manuscript and revising it critically. All authors read and approved the final manuscript.

\section{Authors' information}

All authors had Thai Board of Rehabilitation Medicine. VK is a head of Rehabilitation Medicine Department of Faculty of Medicine Siriraj Hospital, AK is a previous head of Rehabilitation Medicine Department, Faculty of Medicine, Chiang Mai University, SS is a senior staff of Rehabilitation Medicine Department, Phramongkutklao Hospital and College of Medicine, NM is a previous head of Rehabilitation Medicine Department, Faculty of Medicine, KhonKaen University, and YA is a staff of Sirindhorn National Medical Rehabilitation Center, Department of Medical Service, Ministry of Public Health.

\section{Acknowledgements}

The present study was supported by the National Research Council of Thailand, the Clinical Research Collaboration Network, and the Royal College of Physiatrists of Thailand. Authors would like to thank Thai Stroke Rehabilitation Registry (TSRR) Group for their contribution as site coordinators for data collection. We also thank Mr. Suthiphol Udompunturuk and Ms. Julaporn Poolium for statistical analyses.

\section{Author details}

'Rehabilitation Medicine Department, Faculty of Medicine Siriraj Hospital, Mahidol University, 2 Prannok Rd, Bangkok 10700, Thailand. ${ }^{2}$ Rehabilitation Medicine Department, Faculty of Medicine, Chiang Mai University, Chiang Mai, Thailand. ${ }^{3}$ Rehabilitation Medicine Department, Phramongkutklao Hospital and College of Medicine, Bangkok, Thailand. ${ }^{4}$ Rehabilitation Medicine Department, Faculty of Medicine, KhonKaen University, Khon Kaen, Thailand. ${ }^{5}$ Sirindhorn National Medical Rehabilitation Center, Department of Medical Service, Ministry of Public Health, Nonthaburi, Thailand.

Received: 10 December 2012 Accepted: 5 April 2013

Published: 15 April 2013

\section{References}

1. Shah MV: Rehabilitation of the older adult with stroke. Clin Geriatr Med 2006, 22:469-489.

2. Karges J, Smallfied S: A description of the outcomes, frequency, duration, and intensity of occupational, physical, and speech therapy in inpatient stroke rehabilitation. J Allied Health 2009, 38:E1-E10.

3. Doshi VS, Say JH, Young SH, Doraisamy P: Complications in stroke patients: a study carried out at the rehabilitation medicine service, changi general hospital. Singapore Med J 2003, 44:643-652.

4. Dromerick A, Reding M: Medical and neurological complications during inpatient stroke rehabilitation. Stroke 1994, 25:358-361.

5. Kuptniratsaikul V, Kovindha A, Suethanapornkul S, Manimmanakorn N, Archongka Y: Complications during the rehabilitation period in Thai patients with stroke: a multicenter prospective study. Am J Phys Med Rehabil 2009, 88:92-99.

6. Roth EJ, Lovell L, Harvey RL, Heinemann AW, Semik P, Diaz S: Incidence of and risk factors for medical complications during stroke rehabilitation. Stroke 2001, 32:523-529.

7. Domka E, Myjkowska E, Kwolek A: Incidence of neuromedical complications during rehabilitation after stroke. Neurol Neurochir Pol 2005, 39:300-309.

8. Johnston KC, Li JY, Lyden PD, Hanson SK, Feasby TE, Adams RJ, Faught RE $\mathrm{Jr}$, Haley EC Jr: Medical and neurological complications of ischemic stroke: experience from the RANTTAS trial. Stroke 1999, 29:447-453.

9. Kuptniratsaikul V, Kovindha A, Massakulpan P, Piravej K, Suethanapornkul S, Dajpratham P, Manimmanakorn N, Permsirivanich W, Archongka Y, Kuptniratsaikul PS: An epidemiologic study of the Thai stroke rehabilitation registry (TSRR): a multi-center study. J Med Assoc Thai 2008, 91:225-233

10. Mahoney Fl, Barthel DW: Functional evaluation: the barthel index. Md State Med J 1965, 14:61-65.

11. Barczak CA, Barnett Rl, Childs EJ, Bosley LM: Fourth national pressure ulcer prevalence survey. Adv Wound Care 1997, 10:18-26.

12. Bohannon RW, Smith MB: Interrater reliability of a modified Ashworth scale of muscle spasticity. Phys Ther 1987, 67:206-207.

13. World Health Organization: International classification of functioning, disability and health. Geneva: World Health Organization; 2001. 
14. Nilchaikovit T, Lotrakul M, Phisansuthideth U: Development of Thai version of hospital anxiety and depression scale in cancer patients. J Psychiatr Assoc Thai 1996, 41:18-30.

15. Kuptniratsaikul V, Kovindha A, Piravej K, Dajpratham P: A 1-year follow-up of outcomes after stroke rehabilitation: a multi-center study. Biomed Res Int. in press.

16. Pinedo S, de la Villa FM: Complications in the hemiplegic patient in the first year after the stroke. Rev Neurol 2001, 32:206-9.

17. Sackley C, Brittle N, Patel S, Ellins J, Scott M, Wright C, Dewey ME: The prevalence of joint contractures, pressure sores, painful shoulder, other pain, falls, and depression in the year after a severely disabling stroke. Stroke 2008, 39:3329-34

18. Hansen AP, Marcussen NS, Klit H, Andersen G, Finnerup NB, Jensen TS: Pain following stroke: a prospective study. Eur J Pain 2012, 16:1128-36.

19. Lindgren I, Jönsson AC, Norrving B, Lindgren A: Shoulder pain after stroke: a prospective population-based study. Stroke 2007, 38:343-8.

20. Kocabas H, Levendoglu F, Ozerbil OM, Yuruten B: Complex regional pain syndrome in stroke patients. Int J Rehabil Res 2007, 30:33-8.

21. Paci M, Nannetti L, Taiti P, Baccini M, Rinaldi L: Shoulder subluxation after stroke: relationships with pain and motor recovery. Physiother Res Int 2007, 12:95-104

22. Panel O, Khadilkar A, Phillips K, Jean N, Lamothe C, Milne S, Sarnecka J: Ottawa panel evidence-based clinical practice guidelines for post-stroke rehabilitation. Top Stroke Rehabil 2006, 13:1-269.

23. Koyuncu E, Nakipoğlu-Yüzer GF, Doğan A, Ozgirgin N: The effectiveness of functional electrical stimulation for the treatment of shoulder subluxation and shoulder pain in hemiplegic patients: a randomized controlled trial. Disabil Rehabil 2010, 32:560-6.

24. Paci M, Nannetti L, Rinaldi LA: Glenohumeral subluxation in hemiplegia: an overview. J Rehabil Res Dev 2005, 42:557-68.

25. Langhorne P, Stott DJ, Robertson L, MacDonald J, Jones L, McAlpine C, Dick F, Taylor GS, Murray G: Medical complications after stroke: a multicenter study. Stroke 2000, 31:1223-9.

26. Khan M, Ahmed B, Ahmed M, Najeeb M, Raza E, Khan F, Moin A, Shujaat D, Arshad A, Kamal AK: Functional, cognitive and psychological outcomes, and recurrent vascular events in Pakistani stroke survivors: a cross sectional study. BMC Res Notes 2012, 5:89-96.

27. Lee CD, Folsom AR, Blair SN: Physical activity and stroke risk: a metaanalysis. Stroke 2003, 34:2475-81.

28. Kauhanen M, Korpelainen JT, Hiltunen $\mathrm{P}$, Brusin $\mathrm{E}$, Mononen $\mathrm{H}$, Määttä R, Nieminen P, Sotaniemi KA, Myllylä W: Poststroke depression correlates with cognitive impairment and neurological deficits. Stroke 1999, $30: 1875-80$

doi:10.1186/1471-2318-13-33

Cite this article as: Kuptniratsaikul et al: Long-term morbidities in stroke survivors: a prospective multicenter study of Thai stroke rehabilitation registry. BMC Geriatrics 2013 13:33.

\section{Submit your next manuscript to BioMed Central and take full advantage of:}

- Convenient online submission

- Thorough peer review

- No space constraints or color figure charges

- Immediate publication on acceptance

- Inclusion in PubMed, CAS, Scopus and Google Scholar

- Research which is freely available for redistribution 